\title{
Case Report: Unilateral Pulmonary Hypoplasia Presenting as Reccurent Pulmonary Infection
}

\author{
Tri Wahju Astuti ${ }^{1}$, Raden Dicky Wirawan Listiandoko ${ }^{1}$, Garinda Almaduta ${ }^{1}$, Fitri Indah Sari ${ }^{1}$, Dini Rachma \\ Erawati $^{2}$
}

\author{
${ }^{1}$ Department of Pulmonology and Respiratory Medicine, Faculty of Medicine, Brawijaya University, Dr Saiful Anwar General Hospital, Malang, \\ Indonesia, \\ ${ }^{2}$ Department of Radiology, Faculty of Medicine, Brawijaya University, Dr Saiful Anwar General Hospital, Malang, Indonesia
}

DOI: 10.29322/IJSRP.11.12.2021.p12021

http://dx.doi.org/10.29322/IJSRP.11.12.2021.p12021

\begin{abstract}
Pulmonary hypoplasia generally occurs in the neonatal period or in childhood and still exist until adulthood. Pulmonary hypoplasia is characterized by a decrease in the number of bronchial segments and decreased/absent alveolar air spaces. Diagnosis is usually made from history, spirometry, bodyplethysmograph, bronchoscopy, chest x-ray, contrast chest ct scan, chest ct angiography.

Case Report: A 48 year old woman who had been in outpatient treatment for bloody streak cough was taken to referral hospital to establish the disease. In addition, she complaints with dry cough for a week and also accompanied with fever. The diagnose was established from cxr, thorax ct scan, bronchoscopy, thorax ct angiography. From chest $\mathrm{x}$-ray showed a left-sided homogeneous opacity, further imaging chest computed tomography (ct) scan demonstrated a hypoplastic left lung with right lung hyperinflation, review thorax ct scan and the interpretation was left lung hypoplasia, 3D volume-rendered ct image depicts left lung hypoplasia, bronchoscopy from superior and inferior lobe revealed narrowed lumen, chest ct angiography was performed and supports the diagnose of left lung hypoplasia group 3 (Monaldi Classification). The patient had past history of similar respiratory symptoms 20 and 25 years ago and according to her, was treated on both occasions with pneumonia drugs based on chest X-ray findings. No old reports, papers, or investigations were available with the patient and she had remained almost asymptomatic till now. She was gave cefixime 200 twice daily for 5 days, paracetamol, tranexamic acid and vitamin.. Nowadays, she doesn't have respiratory symptoms

Conclusion: Unilateral pulmonary hypoplasia is a rare disease. General treatment is conservative, although surgical resection is indicated in cases of severe cystic changes and severe symptoms
\end{abstract}

Index Terms: Unilateral pulmonary hypoplasia, spirometry, bodyplethysmograph, bronchoscopy, chest x-ray, contrast chest ct scan, chest ct angiography, bronchoalveolar lavage, Candida Spherica

\section{BACKGROUND}

P ulmonary hypoplasia is a relatively uncommon medical condition characterized by incomplete development of the lungs that can affect the overall development of a child. It can be either primary (idiopathic) or secondary to other congenital anomalies and usually leads to severe respiratory insufficiency in pregnancy. This activity describes the pathogenesis, clinical, radiological features and highlights the role of the interprofessional team in evaluating and improving care for patients with this condition (Tisekar et al., 2020). To date, only few cases of pulmonary hypoplasia have been reported. The exact incidence of pulmonary hypoplasia is unknown (Komolafe et al., 2017).

\section{CASE PRESEntation}

A 48-year-old woman was referred to our hospital pulmonary clinic due to a history of bloody cough in June 2019 accompanied with dry cough and fever. The cause of the bloody cough was not identified until the time of the patient's visit to our hospital. There was no respiratory symptoms during this visit. During her first visit in the referring hospital, she was treated with Cefixime $2 \times 200$ for 5 days, Paracetamol 3x500 prn, Tranexamic acid 3x500 mg and Multivitamin 2x1tab. Her CXR showed a suspicion of lung tumor, so she was reffered for Thorax CT scan for further examination to another hospital. CT Scan revealed lung hypoplasia, and thus she was referred to our hospital for further management and treatment.

Physical examination of left hemothorax showed reduced movements, decrease of stem fremitus, dullness on percussion and reduced breath sound and prolonged expiration.

The diagnosis was established from CXR, Thorax CT scan, bronchoscopy, spirometry, bodyplethysmograph, Thorax CT Angiography. Thorax CT Scan is more usefull than bronchography, because it can assess lung hypoplasia, especially for surgery planning (Jeremy et al., 2019). Laboratory results were within normal limit. In lung hypoplasia, blood test is not a specitic parameter, but an increase of leukocytes, or incraese of erythrocyte sedimentation rate (ESR) may be seen in secondary infection (Murrales et al., 2020).

In this case report, there was a secondary infection. Washing From Broncho Alveolar Lavage (BAL) fluid culture afterward showed the growth of Candida spherica (yeast). Blood gas analyze examination can be tested to reveal if there is an oxygen disturbance, which is in this case report there was not found an oxygenation disorder. 


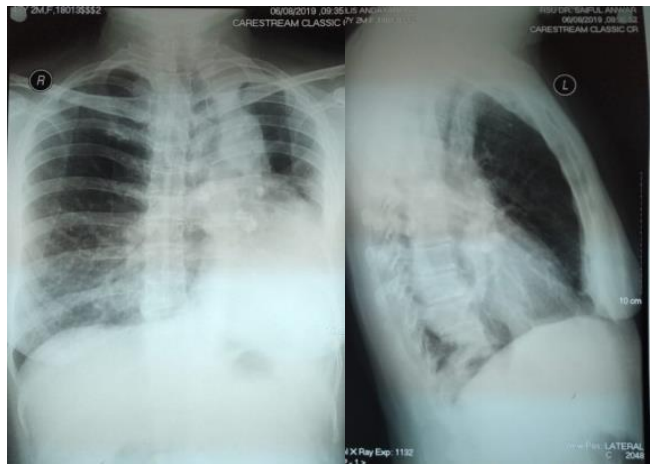

Figure 1. Chest X-Ray at RSSA in August 6, 2019. A. Position PA. B. Position Lateral Sinistra. Showed pneumonia with an atelectasis component

Chest X-ray showed a left-sided homogeneous opacity, shifting of the mediastinum, and chest retraction. The intercostal spaces on the sinistra side were narrowed. Right lung was overinflated (Figure 1). From Chest X-ray suggest of pneumonia with an atelectasis component. Normal lungs generally show overdistention and herniation towards the contralateral hemithorax (Liners et al, 2019). The patient was advised to undergo bodyplethysmograph. Bodyplethysmograph can assest abnormality lung function that have been occured. The results of bodyplethysmograph showed hyperinflation of lung function. Thorax CT scan is an important examination to establish pulmonary hypoplasia which is radiological modality (Figure 2). Further imaging chest computed tomography (CT) scanning demonstrated a hypoplastic left lung with right lung hyperinflation and herniation to the contralateral side, as well as displacement of the mediastinum to the left side.

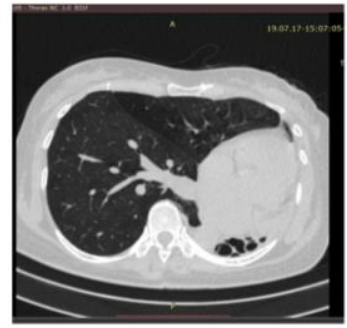

Parenkim Window

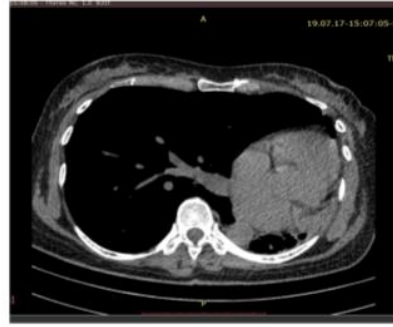

Mediastinal Window
Figure 2. Thorax CT Scan with contrast July 17, 2019 at Persada Hospital. A. Parenchymal window B. Mediastinal window. Revealed left lung hypoplasia with right lung compensatoar hyperinflation

In the diagnose of Destroyed Lung, Lung Contralateral was damaged after tuberculosis and showed residual lesions, such as bronchiectasis, bronchial thickening, calcified granuloma and atelectasis (Porres et al., 2017). Review Thorax CT Scan at RSSA and the interpretation was left lung hypoplasia. 3D volumerendered CT image depicts left lung hypoplasia (Figure 3).

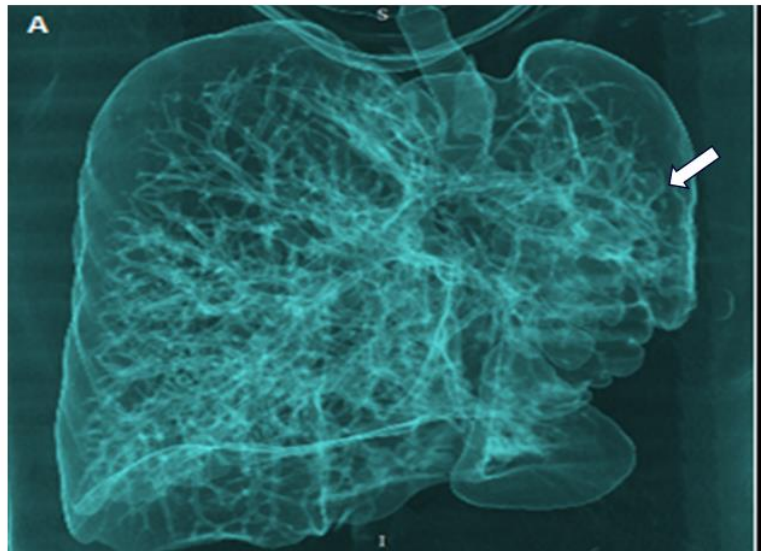

Figure 3. 3D volume-rendered CT image depicts left main bronchus with lobulated dilatation of segmental bronchus. There is absence of left lung parenchymal

Bronchoscopy was performed to observe the endobronchial tree. In this case report, from sinistra superior lobe revealed narrowed lumen, edematous mucosa, and also hyperemia, there is no intraluminal mass, from inferior lobe revealed narrowed lumen, edematous mucosa and also hyperemic, there is no intraluminal mass. In addition, On bronchoscopic examination of the patient has been obtaining samples for cytologic or histopathological examination.

Mycobacterium tuberculosis was not detected in BAL TCM by Genexpert and also was not detected in LJ Media culture. BAL culture grew Candida spherica (yeast). From cytological test (washing and brushing) revealed class II and it was not found malignancies cell. Chest CT angiography (Figure 4) was performed and supports the diagnose of left lung hypoplasia group 3 (Monaldi Classification) accompanied by thickening of the bronchial tree with suspicion of secondary infection.

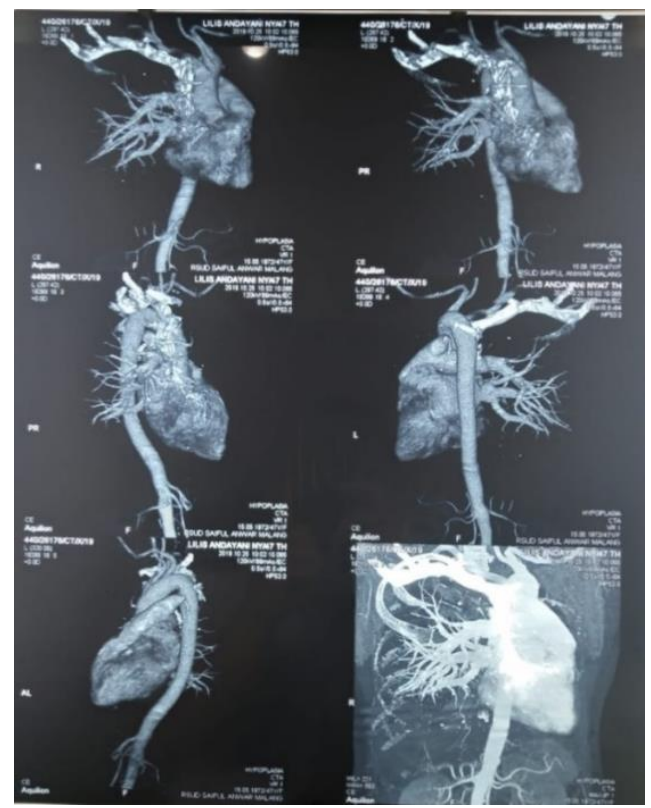

Figure 4. CT Angiography October 25, 2019. Support to establish the diagnose of left lung hypoplasia 
She was treated with oxygen prn, iv plug, ranitidin iv prn, paracetamol tab $3 \times 500 \mathrm{mg}$ prn, codein tab 3x10mg prn. The treatment given to patients during hospitalization on August 6, 2019 to August 10, 2019. She was discharge from RSSA on August 10, 2019 and controlled to Pulmonary Polyclinic for further examination and clinical evaluation. On August 22, 2019, she controlled at Pulmonary Polyclinic at RSSA (Taking the results of Bronchoalveolar Lavage (BAL) Culture: Candida spherica (yeast). Owing to the result from culture was Candida spherica, the doctor gave Fluconazole 1x150mg up to 21 days for antifungal treatment

On October 25, 2019, she was performed thorax CT Angiography. Furthermore, she has never controlled to RSSA because her house was far away (Pasuruan), but clinical evaluations were still carried out by telephone, and the last contact by phone was, at the end of November 2020 where she was no respiratory complaints.

\section{DISCUSSION}

We describe a case of a female 48 year old who was reffered to RSSA for established the diagnose. The symptoms were initially with bloody cough $\pm 0.5-1 \mathrm{ml}$ once, and also dry cough since 1 week ago accompanied with fever. The patient was diagnosed with left lung hypoplasia at RSSA. Lung hypoplasiacor aplasia is a rare condition that is characterized by incomplete development of lung tissue, which can be unilateral or bilateral. It results in a reduction in the number of lung cells, airways, and alveoli that results in impaired gas exchange (Li et al., 2021).

Lung hypoplasia is usually unilateral and occasionally reported to be bilateral (Gulla et al., 2021). Lung hypoplasia is usually secondary to other congenital abnormalities like diaphragmatic hernia, vascular or thoracic cage anomalies, oligohydramnios, maternal treatment with ACE inhibitors, etc. Primary lung hypoplasia presenting in an adult is extremely rare and this is probably the first case reporting its association with a cardiac tumour in an adult. Though no apparent cause is implicated in the pathogenesis of primary lung hypoplasia, it can rarely be associated with cardiac and vascular anomalies such as unilateral absence of the pulmonary artery, cardiac tumours and other congenital heart diseases.

Since these are early errors of development, whether they are cause, effect or an association is difficult to speculate (Mirchandani et al., 2016). In 1912 Schneider classified lung maldevelopment into three groups, modified in 1955 by Boyden as:

a) Type I (Agenesis): complete absence of parenchymal tissue bronchial and vascular supply.

b) Type II (Aplasia): absence of parenchymal tissue but a rudimentary bronchus present, no evidence of pulmonary vasculature.

c) Type III (Hypoplasia): presence of variable amounts of lung parenchyma with decreased number or size of airways, vessels, and alveoli.

Lung hypoplasia was classified in 1960 by Monaldi as:

a) Group 1: No bifurcation of the trachea.

b) Group 2: Only rudimentary bronchus.

c) Group 3: Incomplete development after division of the main bronchus. d) Group 4: Incomplete development of sub segmental bronchi and a small segment of corresponding lobe.

During lung development, the conducting airways are formed first, followed by the formation and enlargement of the gas exchange area. The latter (alveolarization) continues until young adulthood (Schittny 2017). If the pathological process affecting the lung bud occurs during the embryonic stage i.e. up to around $5^{\text {th }}$ week, it results in agenesis, but if it occurs in the later stages it results in aplasia or hypoplasia (Lavina 2016).

Lung hypoplasia is usually encountered in a child presenting with life-threatening symptomatology eg. early respiratory distress after birth, cyanosis, tachypnea, hypoxia, hypercapnia, and acidosis (Zhang et al., 2019). However, it may be infrequently present in adults not producing any symptomatology thus rendering its diagnosis problematic. Nevertheless, some adult patients may present with repeated pulmonary infections and wheezing (Pinheiro et al., 2020).

Autosomal recessive chromosomal aberration, deficiency of vitamin A, intrauterine infections, environmental factors have been held responsible for it (Roy et al., 2012). Physical factors affecting the normal development of the fetal lung is the intrathoracic capacity, the volume of amniotic fluid, the volume and pressure of pulmonary fluid, respiratory movements and blood supply, while transcription and growth factors are involved in the regulation of the process. So, the etiology of Lung hypoplasia may involve space-occupying lesions of the chest; skeletal malformations or large defects in the ab dominal wall that limit the capacity of the rib cage; abnormalities of the urinary tract or nonrenal causes of oligohydramnios that cause loss of lung fluid; neuromuscular or central nervous system abnormalities disrupting fetal breathing movements; cardiac lesions affecting the pulmonary blood supply; and chromosomal abnormalities with a genetic background.

In rare cases, no apparent cause is recognized, so they are referred to as primary (idiopathic) lung hypoplasia. In some of these a familial occurrence has been recognized and the hypothesis of a lack of transcription or growth factors regulating growth of normal lung epithelium, which are possibly inherited in an autosomal recessive mode, has been proposed (Papadopoulos et al., 2018).

Patients usually present in childhood with respiratory distress or recurrent infection or haemoptysis. Rarely they present in adulthood, as in our case. Chest X-ray findings of complete opacification of left hemithorax with volume loss prompt differential diagnoses of suspected foreign body aspiration, mucus plug occlusion, bronchial mass lesions and post infectious collapse. HRCT with CT pulmonary angiography confirm the diagnosis, as in our case. Bronchoscopy in superior and inferior lung sinistra revealed narrowed lumen, edematous mucosa, hyperemia, and also no intraluminal mass.

Pneumothorax and pulmonary hypertension are common serious complications. Pneumothorax often develops spontaneously or secondary to mechanical ventilation in infants who present with severe respiratory insufficiency in the first few hours of life. Last but not least pulmonary infections can cause life-threatening events in patients with this congenital malformation (Katsenos et al., 2014). 
Adult patients with hypoplasia are treated conservatively with antibiotics for infections, symptomatic treatment with bronchodilators and management of complications like haemoptysis. Prophylactic pneumococcal and influenza vaccinations are recommended. Prognosis depends on associated congenital anomalies and the normalcy or otherwise of the other lung (Mirchandani et al., 2016).

Prognosis in those with left sided hypoplasia reported to be better as right lung with its three lobes can minimize the shortcomings by compensatory hyperinflation. Degree of lung and co-organ involvement often influences prognosis. Primary variety has better outcome since patients lack additional abnormalities (Dewan 2015)

\section{CONCLUSION}

Lung hypoplasia is very rare in adulthood. The disease is usually established in the perinatal or neonatal period. Symptomatology is generally present despite the occasional accidental discovery of hypoplastic lung in an asymptomatic adult. Surgical resection of the affected lung is indicated in patients with pronounced symptoms and without severe pulmonary function impairment. In patients with unilateral hypolucent lung, although it is seen rarely, pulmonary hypoplasia should always be kept in mind.

\section{REFERENCES}

[1] Dewan, G., 2015. Unilateral Pulmonary Hypoplasia in a Child. Journal of the Nepal Medical Association, 53(197).

[2] Glasberg T, Jackson P, Pavlova Z, Nair S. Infant with Clinical Evidence of Pulmonary Hypoplasia: A Case Report. Cureus. 2017;9(5):e1298. Published 2017 May 30. doi:10.7759/cureus. 1298

[3] Gulla, K.M., Parihar, M.S., Jat, K.R., Agarwala, S., Lodha, R. and Kabra, S.K., 2021. Congenital Lung Malformations: Experience From a Tertiary Care Center in India. Indian pediatrics, 58(2), pp.129-133.

[4] Katsenos, S., Antonogiannaki, E.M. and Tsintiris, K., 2014. Unilateral primary lung hypoplasia diagnosed in adulthood. Respiratory care, 59(4), pp.e47-e50.

[5] Komolafe, F., Dahniya, M.H., Al-Ali, Y., Al-Zaabi, L. and Aziz, F., 2017. Asymptomatic unilateral pulmonary hypoplasia/agenesis in adults: A report of six cases. West African Journal of Radiology, 24(1), p.99.
[6] Li, J. and Tang, N., 2021. Alveolar stem cells in lung development and regrowth. Lung Stem Cells in Development, Health and Disease (ERS Monograph). Sheffield, European Respiratory Society, pp.17-30.

[7] Mirchandani LV, Alam A, Iyer A, Kutty JT. Rare Case of Unilateral Hypoplasia of Lung with Associated Ventricular Mass in an Adult. J Clin Diagn Res. 2016;10(7):OD05-OD7. doi:10.7860/JCDR/2016/19591.8138

[8] Muralles, I.L., Alarcon-Calderon, A., Najera, S.I.Q. and Mancilla, L.P., 2020. Pulmonary hypoplasia secondary to pulmonary artery stenosis surgical fix for pneumonia?. Chest, 158(4), pp.A415-A416.

[9] Papadopoulos, D., Misthos, P., Chorti, M., Skopas, V., Nakou, A., Karagianidis, N., Lioulias, A. And Filaditaki, V., 2018. Unilateral pulmonary hypoplasia in an adult patient. Monaldi Archives for Chest Disease, 88(1).

[10] Pinheiro G, Alves AM, Rodrigues Neves I, Sequeira T. Left Lung and Pulmonary Artery Hypoplasia: A Rare Case of Hemoptysis. Eur J Case Rep Intern Med. 2020;7(7):001490. Published 2020 Apr 30. doi:10.12890/2020_001490

[11] Porres, D.V., Persiva, O., Pallisa, E. and Andreu, J., 2017. Radiological findings of unilateral tuberculous lung destruction. Insights into imaging, 8(2), pp.271-277.

[12] Schittny, J.C., 2017. Development of the lung. Cell and tissue research, 367(3), pp.427-444.

[13] Tisekar, O.R. and AK, A.K., 2020. Hypoplastic Lung Disease. StatPearls [Internet].

[14] Zhang, L., Ai, T., Wang, L., Zhang, L. and Zhang, Y., 2019. Hypoplasia of the left lung presenting as hemoptysis: A case report. Medicine, 98(4).

\section{AUTHORS}

First Author - Tri Wahju Astuti, Department of Pulmonology and Respiratory Medicine, Faculty of Medicine, Brawijaya University, Dr Saiful Anwar General Hospital, Malang, Indonesia Second Author - Raden Dicky Wirawan Listiandoko, Department of Pulmonology and Respiratory Medicine, Faculty of Medicine, Brawijaya University, Dr Saiful Anwar General Hospital, Malang, Indonesia

Third Author - Garinda Almaduta, Department of Pulmonology and Respiratory Medicine, Faculty of Medicine, Brawijaya University, Dr Saiful Anwar General Hospital, Malang, Indonesia Fourth Author - Fitri Indah Sari, Department of Pulmonology and Respiratory Medicine, Faculty of Medicine, Brawijaya University, Dr Saiful Anwar General Hospital, Malang, Indonesia Fifth Author - Dini Rachma Erawati, Department of Radiology, Faculty of Medicine, Brawijaya University, Dr Saiful Anwar General Hospital, Malang, Indonesia 
International Journal of Scientific and Research Publications, Volume 11, Issue 12, December 2021 